\title{
Modeling the Optical Properties of Excitons in Linear and Tubular J-Aggregates
}

\begin{abstract}
Jasper Knoester
Institute for Theoretical Physics and Materials Science Centre, University of Groningen, Nijenborgh 4, Groningen 9747 AG, The Netherlands
\end{abstract}

Received 18 June 2006; Revised 28 August 2006; Accepted 6 September 2006

\begin{abstract}
The theory of the optical properties of linear and tubular molecular J-aggregates is reviewed. The primary optical excitations in these systems are Frenkel excitons, which may be delocalized over many molecules. The collective nature of these excitations gives rise to special optical properties and dynamics, which are of interest for purely scientific reasons, but also enable the application of J-aggregates as photographic sensitizers and artificial light-harvesting systems. The focus of this paper is on the effect of aggregate geometry, disorder, and temperature on the absorption and fluorescence spectra. Also transport of excitations between J-aggregates is discussed. Connection is made to experiments on aggregates of cyanine dyes and natural light-harvesting systems.
\end{abstract}

Copyright (C) 2006 Jasper Knoester. This is an open access article distributed under the Creative Commons Attribution License, which permits unrestricted use, distribution, and reproduction in any medium, provided the original work is properly cited.

\section{INTRODUCTION}

The study of the collective optical properties of molecular aggregates has had a long history, starting with the pioneering works of Jelley [1] and Scheibe [2] in the 1930s. While continuous progress has been made throughout this history, in particular in relation to color sensitization of photographic emulsions by aggregates of cyanine dyes [3], the scientific interest in these systems has grown strongly in the past 1520 years. The reason lies in (i) novel ultrafast and nonlinear optical techniques that have been applied since the late 1980s to probe the collective optical excitations in aggregates and (ii) the strongly increased theoretical understanding of the many-particle dynamics involved, partly stimulated by the ever growing computational possibilities to simulate these dynamics. Finally, the interest in the optical properties of molecular assemblies has been stimulated by the discovery that light-harvesting complexes in natural photosynthetic systems of bacteria and higher plants are governed by principles very similar to those holding in aggregates of synthesized dyes[4].

During the past two decades, topics of special interest have been the cooperative spontaneous emission of Frenkel excitons in molecular aggregates (exciton superradiance) [5] and the temperature dependence of the emission rate [6-10], the line width $[11,12]$, and energy transport $[13,14]$. For aggregates these properties may exhibit a completely different temperature dependence than for single molecules. Ex- amples are the increase of the radiative lifetime with growing temperature and a nonmonotonic temperature dependence of the fluorescence Stokes shift. The quest for a quantitative understanding of the observed behavior is complicated by the interplay between intermolecular excitation transfer interactions, disorder, and coupling to a phonon bath. Special attention has also been devoted to nonlinear optical response and the role of multiexciton states [15-17]. Two-exciton states may be probed using pump-probe spectroscopy [18], which gives insight into the exciton localization size [19-21]. This size results from the competition between disorder and intermolecular transfer interactions and has been the topic of many studies and much debate, in particular for the photosynthetic antenna complex LH2 of purple bacteria [22].

Varying the aggregate morphology and the effects thereof on the optical response has been another topic of growing interest during the past decade. Triggered by linear dichroism data for J-aggregates of cyanine dyes in a streaming solution [23], until the early 1990s most model studies of molecular aggregates had been performed assuming a linear geometry, that is, treating the aggregate as a one-dimensional object. While many of the optical properties of J-aggregates may indeed be understood using this picture, various types of aggregates have been discovered for which direct imaging reveals an alternative geometry. The best known example is the LH2 complex mentioned above, which has a circular structure, containing two rings of $8-18$ bacteriochlorophyll molecules $[24,25]$. Another example from nature is 
the tubular aggregates that are found in the chlorosomes of green bacteria $[26,27] .^{1}$ The work of Daehne and coworkers, aimed at new classes of carbocyanine dye molecules [29], led to self-assembled systems with a wide variety of geometries $[30,31]$. Amongst those are double-wall cylindrical aggregates [32-34] (coined "amphi-PIPEs"), which in many respects resemble the tubular aggregates in the chlorosomes. It will be of interest to see whether they can be developed into novel types of artificial light-harvesting systems.

The goal of this paper is to present a review of recent advances in modeling the optical properties of Frenkel excitons in J-aggregates, with a specific interest in the role of geometry, disorder, and temperature. This paper closely follows the overview presented at Solar 2006, held in Cairo. The outline of this paper is as follows. In Section 2 the general Frenkel exciton model is presented, the exciton states and absorption spectrum are analyzed for homogeneous linear and tubular aggregates, and the effect of static disorder is discussed. The dynamics of the excitons resulting from the interaction with vibrations is the topic of Section 3, together with the effects of this dynamics on the absorption and fluorescence spectra. In Section 4, we briefly address several aspects of excitation energy transport between molecular assemblies. Finally, in Section 5 we summarize and discuss the outlook of this field.

\section{FRENKEL EXCITONS ON CHAINS AND TUBES}

\subsection{General}

The optical properties of molecular aggregates are governed by Frenkel excitons, that is, excitations in which electron and hole are tightly bound [35]. Driven by intermolecular interactions, the electron-hole pair may propagate through the aggregate, while at each instant of time, electron and hole occupy the same molecule. Frenkel excitons are described conveniently on a basis of molecular excited states. Often it suffices to consider just one such state, which dominates the optical response of the molecule. Let us denote by $|n\rangle$ the aggregate's state in which only molecule $n$ is excited. Linear superpositions of such one-quantum states govern the linear optical properties (absorption and fluorescence) of the aggregate. The corresponding Hamiltonian may be expressed using the basis $\{|n\rangle\}$ and reads:

$$
H_{\mathrm{ex}}=\sum_{n, m} H_{n m}|n\rangle\left\langle m\left|=\sum_{n} \epsilon_{n}\right| n\right\rangle\left\langle n\left|+\sum_{n, m}{ }^{*} J_{n m}\right| n\right\rangle\langle m|
$$

Here, $\epsilon_{n}$ is the transition energy of molecule $n$, which may differ from one molecule to the other as a result of random solvent shifts induced by the host medium. Furthermore, $J_{n m}$ is the excitation transfer interaction between molecules $n$ and $m$, which equals the matrix element of the total electronic interaction Hamiltonian between the molecules calculated for the states $|n\rangle$ and $|m\rangle$ (the asterisk on the summation ex-

\footnotetext{
${ }^{1}$ It should be noted that recently the tubular geometry of these lightharvesting systems has been disputed [28].
}

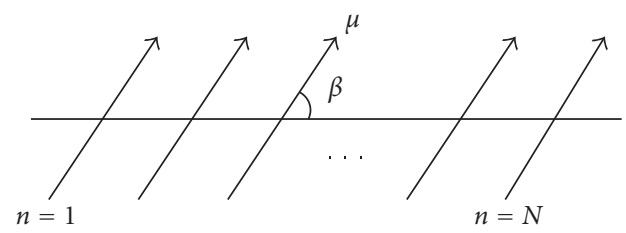

FIGURE 1: Linear aggregate of $N$ equidistant molecules with parallel transition dipoles of magnitude $\mu$. For $\beta<54.7^{\circ}$, the intermolecular dipole-dipole interaction is negative and the chain is a J-aggregate; otherwise it is an $\mathrm{H}$-aggregate.

cludes terms with $n=m$ ) [35]. For large intermolecular separation, $J_{n m}$ reduces to the interaction between the transition point-dipoles of both molecules; for closely spaced molecules it may be necessary to resort to extended dipoles or full integrations over the charge distributions of the molecular states involved [34, 36, 37].

The transition dipole between the ground state and the excited state of molecule $n$ will be denoted $\boldsymbol{\mu}_{n}$. Throughout this paper we will assume that all dipoles have equal magnitude $\mu$, but their directions may differ. This will be particularly important when considering tubular aggregates. The total dipole operator of the aggregate is given by

$$
\mathbf{M}=\sum_{n} \boldsymbol{\mu}_{n}|n\rangle\langle g|+\text { h.c. }
$$

where $|g\rangle$ is the overall ground state, that is, the state in which all molecules are in the ground state. Here, we have assumed that the aggregate (or at least the exciton localization size) is small compared to an optical wavelength.

The exciton Hamiltonian above does not yet account for the interaction with dynamic degrees of freedom in the environment, in particular vibrations. Such interactions, which form the basis for understanding the exciton dynamics and the temperature dependence of the optical properties, will be added in Section 3. At the current level, the model bears most relevance to low-temperature properties.

\subsection{Homogeneous chains and tubes}

Several of the salient optical properties of molecular aggregates may be understood by considering the idealized case in which we neglect disorder. We thus take $\epsilon_{n}=\epsilon_{0}$ for all molecules. The resulting symmetry in the Hamiltonian allows for analytical solutions of the exciton eigenstates.

\subsubsection{Linear aggregates}

We will first consider one-dimensional aggregates. In that case, $n=1, \ldots, N$ labels the positions of the $N$ molecules on the chain and we will assume that all transition dipoles are parallel (see Figure 1). The dipole-dipole transfer interactions are then given by $J_{n m}=-J /|n-m|^{3}$, with $-J$ being the interaction between two nearest neighbors (the minus sign is added, because for J-aggregates the dominant interactions are negative, i.e., $J>0$ ). For simplicity we will assume periodic boundary conditions, identifying molecule $N+1$ with 
molecule 1. In that case, the exciton eigenstates are the Bloch states

$$
|k\rangle=\frac{1}{\sqrt{N}} e^{2 \pi i k n / N}|n\rangle
$$

with energy

$$
E_{k}=\epsilon_{0}-2 J \sum_{n=1}^{(N-1) / 2} \frac{1}{n^{3}} \cos \frac{2 \pi k n}{N},
$$

where $k$ denotes the quantum number (quasimomentum) that takes the values $k=0, \pm 1, \pm 2, \ldots, \pm((N-1) / 2){ }^{2}$

The oscillator strengths of the exciton states are given by

$$
\left|M_{k}\right|^{2}=N \mu^{2} \delta_{k, 0}
$$

This marks the first important result: the $k=0$ state collects all the oscillator strength of the individual molecules; all the other states are dark. This can be traced back to the fact that in the $k=0$ state all molecular dipoles oscillate exactly in phase. Accordingly, this state is referred to as superradiant or as a state with giant oscillator strength.

The superradiant nature of the lowest exciton state in one-dimensional J-aggregates has two consequences. First, the absorption spectrum contains one peak that is redshifted relative to the monomer transition (at $\epsilon_{0}$ ) over an amount $2 J \sum_{n} n^{-3}$, which for long chains equals about $2.4 J$. Such a red-shift indeed is characteristic for J-aggregates and the corresponding peak is referred to as the J-band. This band has been known since the 1930's [1,2]. Second, since the spontaneous emission rate of a state is proportional to its oscillator strength, the $k=0$ state shows a radiative decay that is $N$ times faster than that of a single molecule. Such ultrafast spontaneous emission was indeed observed for the first time by Wiersma and coworkers, for aggregates of the dye pseudo-isocyanine (PIC) [5].

The gathering of most of the oscillator strength in one state is not limited to aggregates with periodic boundary conditions. Even if we apply open boundary conditions (which break the perfect translational symmetry), we find that the lowest exciton state acquires as much as $83 \%$ of the oscillator strength and for long chains is positioned at the same energy as with periodic boundary conditions [38]. To close this section, we note that also in two-dimensional aggregates (molecular monolayers, such as those formed by the Langmuir-Blodgett technique), one absorption peak dominates, provided that the transition dipoles of all molecules are parallel to each other [39]. As we will see, for tubular aggregates (rolled monolayers), more than one exciton state becomes superradiant.

\footnotetext{
2 It is assumed that $N$ is odd; if $N$ is even, we have $k=0, \pm 1, \pm 2, \ldots$, $\pm(N / 2-1), N / 2$ and the summation over $n$ in (4) extends over $n=$ $1,2, \cdots,(N / 2-1)$ and adds the term with $n=N / 2$ with an overall factor $1 / 2$. Similar for $k_{i}$ and $s_{i}$ in the case of the tubular aggregate.
}

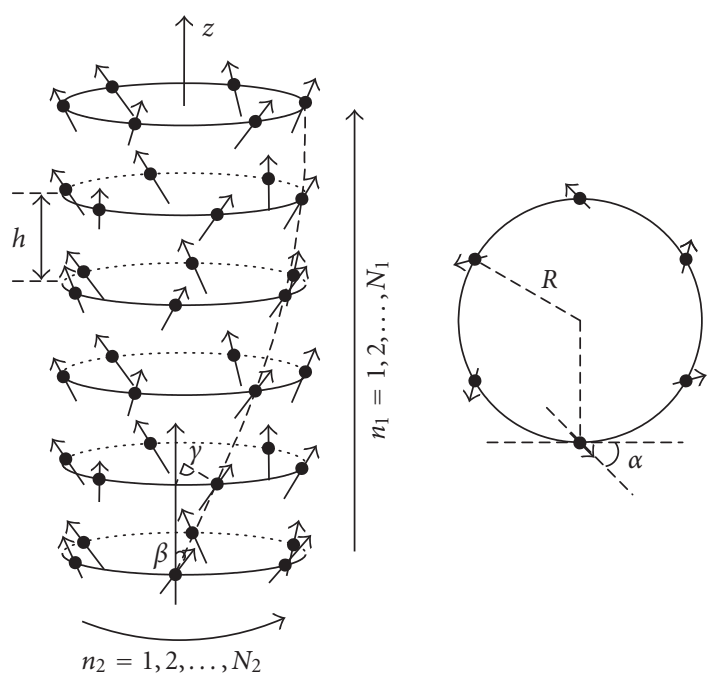

FIgURE 2: Cylindrical aggregate consisting of a stack of $N_{1}$ rings, labeled $n_{1}=1,2, \ldots, N_{1}$, such that each contains $N_{2}$ molecules labeled $n_{2}=1,2, \ldots, N_{2}$. The arrows indicate the transition dipoles. Each ring is rotated with respect to the previous one over an angle $\gamma$, giving rise to a helical structure.

\subsubsection{Tubular aggregates}

As mentioned in the Introduction, tubular molecular aggregates occur in the photosynthetic systems of green bacteria and also have been prepared synthetically using amphiphilic cyanine dyes. Both the natural and synthetic systems have diameters in the order of $10 \mathrm{~nm}$ and a length in the order of a few hundred nanometers, up to microns. As we will see, the optical properties of tubular aggregates are richer than those of linear chains (and monolayers), in the sense that they have more than one superradiant state and more than one J-band.

A tubular aggregate consisting of a single wall may be thought of as a two-dimensional lattice of molecules that is wrapped on a cylindrical surface without a seam [34], much like the construction of a carbon nanotube from a graphene sheet. Alternatively, it can be shown that such an aggregate may be considered a stack of rings, as has been illustrated in Figure 2 [34]. There are $N_{1}$ equidistant rings stacked on top of each other, with each ring containing $N_{2}$ equidistant molecules. In case the tube has a helical structure, each next ring is rotated relative to the previous one over an angle $\gamma$. Each molecule is uniquely labeled by a two-dimensional vector $\mathbf{n}=\left(n_{1}, n_{2}\right)$, where $n_{1}$ denotes the ring number and $n_{2}$ the position of the molecule in that ring. The transition dipoles of the molecules follow the cylindrical symmetry. Thus, the component of the dipole along the axis of the cylinder is equal $(\mu \cos \beta)$ for each molecule, while the component in the plane of the rings has equal magnitude $(\mu \sin \beta)$ and rotates around the cylinder with a constant angle $\alpha$ relative to the local tangent of the ring at the position of the molecule.

In the absence of disorder, the system has cylindrical symmetry which dictates Bloch character of the exciton eigenstates in the $n_{2}$ coordinate. If we assume that we are dealing with long cylinders, it is reasonable to impose 
periodic boundary conditions in the $n_{1}$ direction with $N_{1} \gamma=$ $2 \pi l$ ( $l$ integer), which implies that also in that coordinate the eigenstates have Bloch nature. We then have as exciton states

$$
|\mathbf{k}\rangle=\left|\left(k_{1}, k_{2}\right)\right\rangle=\frac{1}{\sqrt{N_{1} N_{2}}} \sum_{\mathbf{n}} e^{2 \pi i\left[k_{1} n_{1} / N_{1}+k_{2} n_{2} / N_{2}\right]}|\mathbf{n}\rangle,
$$

with $k_{i}=0, \pm 1, \pm 2, \ldots, \pm\left(\left(N_{i}-1\right) / 2\right)(i=1,2)$ (see footnote $2)$. The energy of these states is given by

$$
E_{\mathbf{k}}=\epsilon_{0}+2 \sum_{s_{1}=0}^{\left(N_{1}-1\right) / 2} \sum_{s_{2}=0}^{\left(N_{2}-1\right) / 2} * J(\mathbf{s}) \cos \left(2 \pi\left[\frac{k_{1} s_{1}}{N_{1}}+\frac{k_{2} s_{2}}{N_{2}}\right]\right)
$$

where $\mathbf{s}=\left(s_{1}, s_{2}\right), J(\mathbf{s})=J_{\mathbf{n}+\mathbf{s}, \mathbf{n}}$ and the asterisk excludes the term $\mathbf{s}=\mathbf{0}$.

For long cylinders, the oscillator strengths of the collective states are given by [40],

$$
\left|M_{\mathbf{k}}\right|^{2}=N_{1} N_{2} \mu^{2}\left[\cos ^{2} \beta \delta_{\mathbf{k}, \mathbf{0}}+\frac{1}{2} \sin ^{2} \beta\left(\delta_{\mathbf{k}, \mathbf{h}}+\delta_{\mathbf{k},-\mathbf{h}}\right)\right],
$$

with $\mathbf{h}=\left(\gamma N_{1} / 2 \pi, 1\right)$, the helical wave vector. Hence, there are three superradiant states; the one with $k_{2}=0$ is polarized along the axis of the cylinder, while those with $k_{2}= \pm 1$ are polarized perpendicular to this axis, that is, in the plane of the rings. The reason is that for $k_{2}=0$ the components of the molecular dipoles along the axis add in phase to form a giant dipole $\left(\sim \sqrt{N_{1} N_{2}}\right)$, while those in the planes of the rings add to zero. For the $k_{2}= \pm 1$ states, the components along the axis, weighted with the appropriate Bloch factor, add to zero, while the components in the plane of the rings add to form giant dipoles.

The states with $\mathbf{k}= \pm \mathbf{h}$ are degenerate, so that together they give rise to one absorption peak. Hence, the absorption spectrum of a long cylinder has two peaks with mutually perpendicular polarization. The positions of these peaks are determined by the system parameters, in particular they are strongly affected by the orientation of the dipoles and the relative position of nearest-neighbor molecules in adjacent rings. In the case of the double-wall cylinders formed by self-assembly of the dye 3, $3^{\prime}$-bis(3-sulfopropyl)-5, 5' , 6, 6' tetrachloro-1, 1' -dioctylbenzimidacarbocyanine (C8S3) and also for the chlorosomes in green bacteria, the superradiant state with $\mathbf{k}=\mathbf{0}$ lies at the lower edge of the band of states with $k_{2}=0$, while the states with $\mathbf{k}= \pm \mathbf{h}$ lie at a finite distance above the edge of the $k_{2}=1$ band.

This basic picture agrees with the absorption and linear dichroism (LD) spectra of the C8S3 amphi-PIPEs, for which three J-bands (red-shifted relative to the molecular transition) are observed, with the lower two polarized along the tube axis and the highest perpendicular to it [34]. As these aggregates contain two weakly interacting concentric cylinders (double wall system), one expects four J-bands: two polarized parallel and two perpendicular to the axis. It turns out that the superradiant states with $\mathbf{k}= \pm \mathbf{h}$ are so close in energy for the two cylinders, that their absorption peaks (broadened by disorder and dynamics) merge into one. This is confirmed by pump-probe experiments, which show that the highest energy J-band indeed can be associated with both cylinders $[41,42]$. For chlorosomes, which have a singlewall cylindrical structure, the superradiant transitions in the $k_{2}=0$ and $k_{2}= \pm 1$ bands are separated by less than their widths, which makes it hard to distinguish them in absorption and linear dichroism experiments [27, 40]; the circular dichroism reveals a rich structure, however, which can also be understood on the basis of the $k_{2}=0, \pm 1$ bands [40].

While for a system without disorder the Bloch nature of the states in the $n_{2}$ direction is exact (owing to the cylindrical symmetry), the use of periodic boundary conditions and Bloch wave functions in the $n_{1}$ direction is really limited to long cylinders. It turns out that for the absorption and LD spectra this length limitation is not too severe [43]. By contrast, for calculating the circular dichroism (CD) spectrum, the use of periodic boundary conditions is very restrictive. The reason is that this spectrum is very sensitive to small differences in transition energies and rotary strengths. Analytical solutions that circumvent this problem first use the decomposition into bands characterized by a transverse wave number $k_{2}$ (same meaning as above) and then solve the effective one-dimensional problem left for each $k_{2}$ band. Then dipole allowed states only exist in the $k_{2}=0$ band (polarized along the cylinder axis) and in the $k_{2}= \pm 1$ bands (degenerate and polarized perpendicular to the axis). Good approximate solutions to the remaining one-dimensional problems have been found, which show that in the $k_{2}=0$ band one superradiant state dominates the optical response, while in the $k_{2}= \pm 1$ bands three close lying states dominate [43]. These three states exhibit a strongly helical distribution of exciton amplitude on the cylinder. For very long cylinders, they get degenerate at the energy $E_{\mathbf{h}}$, so as to become consistent with the solution with periodic boundary conditions.

\subsection{Effects of disorder}

Energetic disorder breaks the translational symmetry and mixes the exciton eigenstates found in the absence of disorder. As a result, excitons that are (almost) dark in the absence of disorder and that lie close to a superradiant state will borrow oscillator strength from this state. For one-dimensional J-aggregates this leads to the typical asymmetric lineshape of the J-band at low temperatures (cf. Figure 3). As the superradiant state lies at the bottom of the homogeneous band, it only mixes with higher-lying states, resulting in a highenergy tail of the absorption band. The mixing with the higher states also pushes the J-band down in energy, leading to a disorder-induced red-shift. Generally, one resorts to numerical simulations to deal with disordered aggregates $[38,44]$, but other methods, such as the coherent potential approximation, also give excellent results for linear optical spectra $[39,45]$.

Mixing of the states leads to their localization on parts of the system $[38,44,46]$. As the superradiant emission rate depends on the number of molecules that are excited in phase, it is proportional to the typical band-edge exciton localization size, in stead of the total aggregate size. This explains why the low-temperature superradiant emission rate of linear PIC 


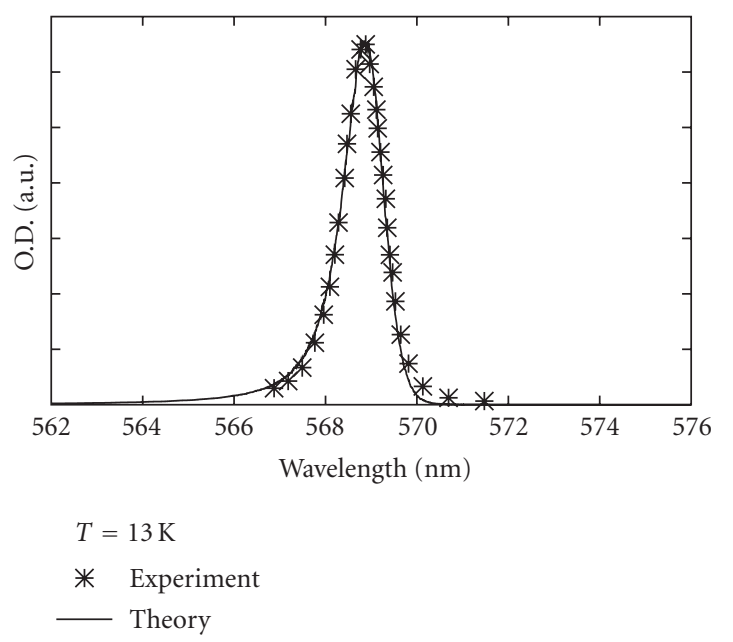

FIGURE 3: Simulated J-band for linear J-aggregates of 500 molecules with static disorder of strength $\sigma=0.13 J$ and $J=600 \mathrm{~cm}^{-1}$ (solid line), compared to the measured J-band for aggregates of PIC-Cl at $13 \mathrm{~K}$.

aggregates is only $50-70$ times larger than that of a single molecule $[5,7,8]$, even though these aggregates have a length of thousands of molecules. The excitons apparently are localized on $50-70$ molecules. This is confirmed by numerical simulations of the exciton Hamitonian (1) accounting for uncorrelated Gaussian disorder in the molecular transition energies $\epsilon_{n}$, which gives a good fit of the low-temperature Jband for a disorder strength (standard deviation of the Gaussian distribution) $\sigma=0.13 \mathrm{~J}$ in the case of PIC-Cl [47]. At this value of the disorder, the typical localization size of the excitons at the lower band edge, $N^{*}$, calculated from the so-called participation ratio $[38,44]$, is in the order of 60 molecules, which nicely agrees with the emission rate.

In addition to its red-shift relative to the monomer spectrum and its asymmetric shape, there is a third characteristic property of the J-band, namely, its narrowness $[1,2]$. This derives from the fact that the exciton averages over the uncorrelated energy disorder of the individual molecules within its localization domain and leads to a $\sigma / \sqrt{N^{*}}$ scaling of the J-band width at low temperatures. This effect is called motional or exchange narrowing of the disorder [48]. Using numerical as well as self-consistent analytical arguments, it may be shown that for one-dimensional aggregates this leads to a $\sigma^{4 / 3}$ scaling of the J-band width at low temperatures $[38,44,46]$.

While the above discussion of disorder was mostly illustrated with the case of linear J-aggregates, asymmetric lineshapes, exciton localization, and exchange narrowing are associated with disordered tubular aggregates as well. In fact the absorption and LD spectra of the C8S3 double-wall amphi-PIPEs have been explained at a quantitative level using a model that accounts for energy disorder [34]. The disorder leads to broadening and some overlap between the superradiant states of both walls, but still it is possible to associate the three experimentally observed J-bands with the superradiant states distinguished in Section 2.2.2. An inter- esting new aspect compared to linear aggregates is that the exciton localization may be anisotropic on the surface of the cylinder, which may be visualized by plotting the autocorrelation function of the exciton wave function [45]. For helical cylinders, this quantity clearly reveals anisotropic localization properties.

\section{TEMPERATURE DEPENDENCE}

As mentioned in the introduction, the temperature dependence of the optical properties of J-aggregates shows several interesting anomalous aspects, such as a radiative lifetime that grows with temperature [7-9] and a nonmonotonic Stokes shift [12]. Also the temperature dependence of the absorption line width has been the topic of several studies $[6,11,49]$. Temperature dependence arises from a coupling of the excitons to a thermal bath, in particular to vibrations in the aggregate and (or) in the host medium. This coupling arises from the fact that displacements in the environment of a particular molecule lead to changes in its transition energy. If one expands this energy shift in powers of the displacements, the lowest-order coupling may be written $[35,50]$,

$$
V=\sum_{n=1}^{N} \sum_{q} V_{n q}|n\rangle\langle n| a_{q}+\text { h.c. }
$$

where $q$ labels the vibrational modes, $V_{n q}$ characterizes the coupling of mode $q$ to an excitation on molecule $n$, and $a_{q}$ $\left(a_{q}^{\dagger}\right)$ annihilates (creates) a vibration in mode $q$. The total Hamiltonian of the system of interest is obtained by summing the bare exciton Hamiltonian $H_{\mathrm{ex}}$ of (1), the interaction $V$, and the bare vibration Hamiltonian given by

$$
H_{\mathrm{vib}}=\sum_{q} \omega_{q} a_{q}^{\dagger} a_{q},
$$

where $\omega_{q}$ denotes the energy of a vibration in mode $q$ (we set $\hbar=1$ ). In this paper, we will restrict ourselves to the above linear exciton-vibration coupling. The next, quadratic, order of the expansion contains interaction terms of the form $V_{n q q^{\prime}}^{(2)}|n\rangle\langle n|\left(a_{q}+a_{q}^{\dagger}\right)\left(a_{q^{\prime}}+a_{q^{\prime}}^{\dagger}\right)$, which will be neglected here [51]. Furthermore, motivated by the disordered nature of most host media that are used in experimental studies of aggregates, we will treat the coupling parameter $V_{n q}$ as a stochastic variable with respect to the site index $n$, characterized by $\left\langle V_{n q}\right\rangle=0$ and $\left\langle V_{m q} V_{n q}^{*}\right\rangle=\delta_{m n}\left|V_{q}\right|^{2}[50]$.

If the exciton-vibration coupling is not too large, it is convenient to express $V$ on the basis of eigenfunctions of the bare exciton Hamiltonian $H_{\mathrm{ex}}$ and treat it perturbatively. The exciton eigenfunctions in the presence of disorder will be labeled $|\nu\rangle$. They read

$$
|\nu\rangle=\sum_{n} \varphi_{\nu n}|n\rangle,
$$

with $\varphi_{v n}$ being the $n$th element of the $\nu$ th eigenvector of the matrix $H_{n m}$ defined in (1). The corresponding eigenvalue $E_{v}$ denotes the exciton energy. Treated as a perturbation, the exciton-vibration coupling then leads to transfer of excitation probability from one localized state, $|\nu\rangle$, to another, $|\mu\rangle$. 
Using Fermi's golden rule, the rate for this transfer, denoted by $W_{\mu \nu}$, is obtained as [50],

$$
W_{\mu \nu}=\mathcal{F}\left(\left|\omega_{\mu \nu}\right|\right) G\left(\omega_{\mu \nu}\right) \sum_{n}\left|\varphi_{\mu n} \varphi_{\nu n}\right|^{2}
$$

Here, $\omega_{\mu \nu}=E_{\mu}-E_{v}$ and $\mathcal{F}(\omega)=2 \pi \sum_{q}\left|V_{q}\right|^{2} \delta\left(\omega-\omega_{q}\right)$, which is known as the one-phonon spectral density. Furthermore, $G(\omega)=n(\omega)$ if $\omega>0$ and $G(\omega)=1+n(-\omega)$ if $\omega<0$, with $n(\omega)=\left[\exp \left(\omega / k_{B} T\right)-1\right]^{-1}$, the mean thermal occupation number of a phonon mode of energy $\omega$. We draw attention to the fact that this is where temperature enters our model. Equation (12) holds for any system geometry and disorder realization. We also observe that $W_{\mu \nu}$ is proportional to the overlap $\sum_{n}\left|\varphi_{\mu n} \varphi_{\nu n}\right|^{2}$ of the excitation probabilities of both excitons, which implies that transfer does not occur between states localized on different parts of the aggregate.

The rates $W_{\mu \nu}$ describe the population dynamics within the exciton band (intraband relaxation), which have a direct effect on the spectral and temporal characteristics of the fluorescence. We will come back to this later in this section. We first notice, however, that the population transfer also gives rise to a lifetime-induced dephasing rate, which for the $\nu$ th exciton state is given by $\Gamma_{\nu} \equiv(1 / 2) \sum_{\mu} W_{\mu \nu}$ and depends on temperature through the $n(\omega)$. Together with the dephasing rate $\gamma_{v} / 2$ caused by spontaneous emission to the ground state, this thermal dephasing rate determines the homogeneous line width $\Gamma_{\nu}+\gamma_{v} / 2$ that is associated with the contribution of the exciton $|\nu\rangle$ to the absorption spectrum.

Using the above, one may simulate the absorption spectrum as a function of temperature, accounting for static disorder as well as coupling to vibrations [47],

$$
A(E)=\left\langle\sum_{\nu} \frac{F_{v}}{\pi} \frac{\Gamma_{\nu}+\gamma_{\nu} / 2}{\left(E-E_{\nu}\right)^{2}+\left(\Gamma_{\nu}+\gamma_{\nu} / 2\right)^{2}}\right\rangle,
$$

where $F_{v}=\left(\sum_{n} \varphi_{v n}\right)^{2}$ is the dimensionless oscillator strength of the $v$ th exciton state, $\gamma_{\nu}=\gamma_{0} F_{v}$ is its radiative rate ( $\gamma_{0}$ is the emission rate of a single chromophore), and the angular brackets denote averaging over the static disorder in the $\epsilon_{n}$. In order to calculate this spectrum, a choice has to be made for the spectral density $\mathcal{F}(\omega)$. As in general one lacks detailed information about the vibrational modes and their coupling to the molecular excitations, one mostly resorts to a phenomenological form. Common choices follow a power law in $\omega$, with a possible exponential cut-off at high frequencies.

In the study of linear aggregates of PIC, several authors have addressed the exciton-phonon coupling in order to model temperature dependent experiments. Mostly, vibrations of the chains themselves have been considered; often these were high-frequency local modes $[6,10,49]$. However, in the case of a linear aggregate in a host medium, especially at temperatures that are not too high (up to about $100 \mathrm{~K}$ ), it seems more reasonable to assume that the coupling to
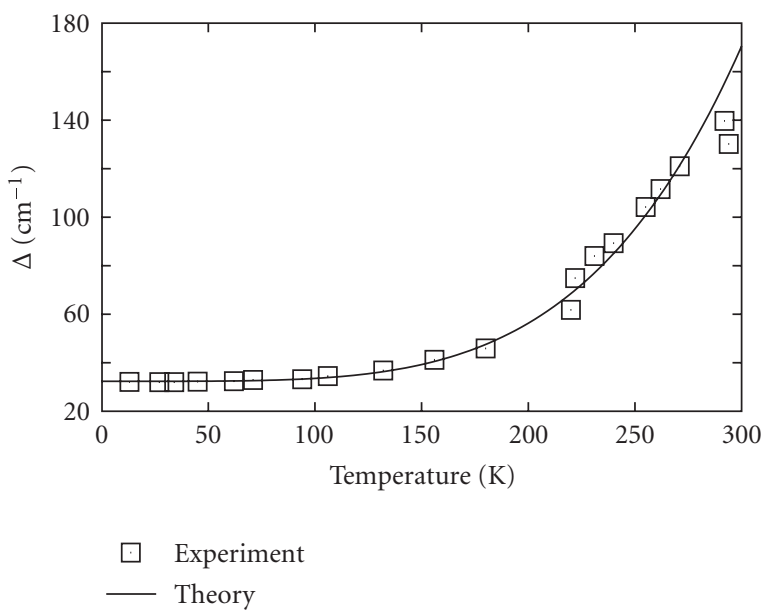

FIGURE 4: Simulated (solid line) and measured (squares) full width at half maximum of the J-band of PIC-Cl aggregates as a function of temperature. Simulation parameters were $\sigma=0.13 \mathrm{~J}$ and $W_{0}=$ $16.0 J$ with $J=600 \mathrm{~cm}^{-1}$.

acoustic phonons in the host medium dominates the exciton dynamics. This leads to a spectral density that scales as $\mathcal{F}(\omega)=W_{0}(\omega / J)^{3}$, with $W_{0}$ being a phenomenological parameter that denotes the overall scattering amplitude in the model $[47,51]$.

Extensive numerical simulations of the absorption lineshape of this model for linear aggregates coupled to acoustic phonons have given several interesting results [47, 51]. First, not surprisingly, the line width increases with temperature and the J-band becomes more symmetric, because homogeneous broadening becomes increasingly more important. Second, the full width at half maximum follows an almost universal power law: $\Delta(T)=\Delta(0)+a W_{0}\left(k_{B} T / J\right)^{p}$, where $a$ and $p$ depend only weakly on the parameters $\sigma$ and $W_{0}$. This scaling relation implies that, although the spectrum results from a distribution of exciton states with different dephasing rates, the total width may be interpreted as the sum of an inhomogeneous width, $\Delta(0)$, and a dynamic contribution. Third, it turned out that the above model (with the disorder strength $\sigma$ and the scattering strength $W_{0}$ as only fit parameters) yields excellent quantitative fits of absorption and hole-burning line width measurements for PIC aggregates with a variety of counter ions for temperatures up to room temperature (see Figure 4 for PIC-Cl). This strongly suggests that acoustic phonons in the host medium play an essential role in the exciton dynamics. It should be noted that a linear frequency scaling of $\mathcal{F}(\omega)$ cannot describe the experiments [51].

We now turn to the exciton fluorescence. In these experiments, one usually excites the system high in the exciton band. The vibration-induced scattering rates $W_{\mu \nu}$ lead to relaxation of the exciton population in this band. Finally, near the band bottom, where the states with most oscillator strength reside, the exciton may decay radiatively with rate $\gamma_{\nu}$. These various processes are shown schematically in 


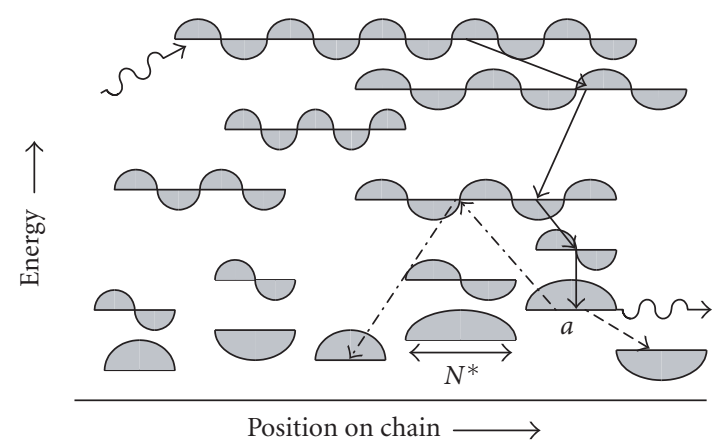

FIGURE 5: Schematic picture of the localized exciton wave functions near the lower band edge in a disordered linear chain. Also indicated are the optical pumping and emission processes (wavy arrows) and various intraband relaxation processes (straight arrows).

Figure 5 and are described mathematically by the Pauli master equation $[52,53]$,

$$
\dot{P}_{\nu}=R_{\nu}(t)-\left[\gamma_{\nu}+\sum_{\mu} W_{\mu \nu}\right] P_{\nu}+\sum_{\mu} W_{\nu \mu} P_{\mu} .
$$

Here, $R_{v}(t)$ describes a (pulsed) excitation source. Solution of this equation yields all the ingredients to determine the time dependent fluorescence spectrum as well as the integrated fluorescence intensity as function of time.

Both the spectrum and the intensity decay sensitively depend on the interplay between relaxation and spontaneous emission. It is particularly important whether the population distribution reaches thermal equilibrium over the exciton band prior to emission or not. If it does, we are in the limit of fast relaxation and the situation is most intuitive. In this case the Stokes shift of the fluorescence spectrum monotonically decreases with growing temperature, indicating the upward shift of the mean energy of the equilibrium population distribution. However, at low temperatures, the transfer rates diminish due to the smallness of the phonon occupation numbers $n(\omega)$. Moreover, at the bottom of the exciton band, the states are most strongly localized, leading to small spatial overlap integrals between the bottom states of different localization segments. As a result, at low temperatures the equilibration near the band bottom slows down dramatically, so that the exciton may emit from a nonequilibrium state [53]. For instance, it may be trapped in and emit from state $a$ in Figure 5, even if states in neighboring segments are lower in energy by an amount $k_{B} T$ or more. When temperature increases slightly, the possibility for the exciton in $a$ to reach a lower state in its neighborhood increases; this may happen by making an intermediate transfer step via a higher-energy state that overlaps with both segments (dash-dotted lines in Figure 5). Thus, with increasing temperature the probability to emit from a lowerenergy state may in fact increase. This shows that at low temperature the Stokes shift may increase with growing temperature. This phenomenon has been observed for linear
J-aggregates of the dye 3, $3^{\prime}$-bis(sulfopropyl)-5, $5^{\prime}$-dichloro9-ethylcarbocyanine (THIATS), as well as for the C8S3 tubular aggregates. In both cases quantitative fits have been found using the above level of modeling $[53,54]$.

We finally turn to the decay of the fluorescence intensity after pulsed excitation. If we assume equilibration on the time scale of the emission, one-dimensional J-aggregates, with all the optically active states at the bottom of the exciton band, exhibit an ultrafast decay at low temperatures that is enhanced by the typical band edge localization size $N^{*}$ (Section 2.3). With increasing temperature, population will be redistributed from the low-lying superradiant states to the (practically) dark states higher in the exciton band. This leads to a reduction of the oscillator strength per populated state, which in turn results in a slowing down of the decay. This thermal destruction of exciton superradiance indeed has been observed for a variety of molecular aggregates [6-9] and at low temperatures it may be well described on the basis of the exciton population dynamics governed by the Pauli master equation $[47,53]$. The generic behavior in this regime seems to be an approximate linear scaling with $T$.

For higher temperatures, however, this level of description breaks down, because the scattering of the excitons on the vibrations becomes so strong that the coherence length of the excitation is no longer limited by the exciton localization length $N^{*}$ imposed by disorder, but rather by the scattering on the vibrations. The perturbative approach, which was based on the exciton states $|\nu\rangle$ and led to (14), then looses its validity. This situation is of particular relevance to the case of PIC aggregates, whose lifetime increases steeply with temperature above about $50 \mathrm{~K}$. Using physical arguments, it has been possible to relate the exciton coherence length imposed by scattering on vibrations to the thermal broadening of the absorption band [47], leading to a quantitative fit of the steep rise of the lifetime. This fit demonstrates that it is not necessary to resort to a higher-dimensional geometry for PIC aggregates in order to understand the temperature dependence of its fluorescence lifetime [8]. We note in passing that at least up to temperatures of $100 \mathrm{~K}$ aggregates of the dye THIATS do not show a similar steep rise of their lifetime $[12,53]$. This may be due to the fact that for these aggregates $N^{*} \approx 30$, which is considerably smaller than for PIC $\left(N^{*} \approx 60\right)$, so that the temperature where the coherence length falls below $N^{*}$ is higher than for PIC.

It should be noted that at low temperatures the fluorescence decay generally is nonexponential, even if this decay is spectrally resolved. The reason is that due to the static disorder at each given energy a broad distribution occurs for the oscillator strength per state at that energy [55]. The decay becomes exponential only when the exciton populations thermalize before emission, that is, at elevated temperatures. Nevertheless, even in the absence of complete thermalization, one still observes an increase of the typical decay time (such as the 1/e time) of the integrated fluorescence intensity with growing temperature [55]. We finally note that in the case of tubular aggregates, the existence of more than one Jband gives rise to interesting aspects of equilibration not only within each band, but also between different bands [54]. 


\section{EXCITON TRANSPORT BETWEEN AGGREGATES}

Incoherent excitation energy transfer between single molecules is an important and well-known phenomenon. The standard Förster formalism treats the intermolecular dipoledipole interaction perturbatively (Fermi's golden rule) and yields a transfer rate that is inversely proportional to the sixth power of the distance and directly proportional to the overlap of the donor emission and the acceptor absorption spectrum [56]. Only dipole-allowed transitions contribute. Recently, the analog of Förster transfer between closely spaced molecular aggregates has been the topic of many investigations. Much of this research was triggered by studies of excitation transfer between the two rings of the LH2 complex in purple bacteria [57-60]. For the double-wall C8S3 aggregates, energy transport of excitation from one wall to the other has been observed as well. It appears from steady-state fluorescence experiments that even at temperatures as low as $5 \mathrm{~K}$, this transport is fast enough to compete with the spontaneous emission [54]. Ultrafast pump-probe experiments at room temperature reveal that the transfer time scale is in the order of 300 femtoseconds [42].

Excitation energy transfer between two closely spaced aggregates is a fundamentally different problem than the classical Förster situation. First, due to the size of the aggregates, the sum of the dipole-dipole transfer interactions between their molecules does not reduce to the effective interaction between the transition dipoles of their exciton states (unless the separation is large compared to the aggregate size, in which case the energy transfer is very slow anyhow). As a consequence, also dark states contribute to the energy transfer between aggregates, implying that the overlap integral of emission and absorption spectrum does not suffice to determine its rate. This was first realized by Sumi [57].

A second new aspect in the energy transfer between aggregates as compared to single molecules is that aggregates have a whole band of closely lying states. As a result the energy transfer competes with intraband relaxation within each of the aggregates, leading in general to a strongly nonexponential transfer process. This makes it hard to define a unique transfer rate, unless the intraband relaxation is much faster than the energy transfer [61].

Finally, the third difference also is related to the fact that we deal with exciton bands. The bands of the donor and the acceptor aggregate may overlap. This, for instance, is the case for the inner and outer cylindrical walls of the C8S3 aggregates. As a consequence, many near-degeneracies occur between exciton states of both aggregates. Depending on the homogeneous line width of the exciton states, this may invalidate a perturbative treatment of the interactions between the aggregates. Instead, the exciton bands of both aggregates mix, and the transfer problem becomes part of a complicated relaxation process within the new mixed band. The effects of intraband relaxation and band mixing were analyzed in detail for two parallel linear J-aggregates with different exciton band edges, but appreciable overlap of both bands [61]. This serves as model situation for the interacting inner and outer walls of the C8S3 aggregates. It was found in particular that with increasing temperature, the validity of the perturbative approach deteriorates, which is a consequence of the fact that near-degeneracies are more common for the higher-energy parts of the exciton bands.

\section{CONCLUSIONS AND OUTLOOK}

In this paper a brief review has been given of the models used to describe the optical dynamics in molecular aggregates. The starting point was the Frenkel exciton model, to which static energetic disorder and interactions with a bath of vibrations were added. Other extensions of the model are possible. For instance, we may add disorder in the transfer interactions as well as the modulation of these interactions due to vibrations. The former gives rise to more symmetric low-temperature J-bands than observed in experiment [38]; the latter does not lead to fundamentally new phenomena and only introduces an additional free parameter that may be used to fit experiments [47]. As discussed, the model presented here has been used to generate good fits for the temperature dependence of a variety of optical properties, for several types of aggregates, both linear and tubular.

One of the challenges for the near future is the understanding and control of exciton motion within and between aggregates. In order to use synthetic aggregates as artificial light-harvesting systems, it is necessary to optimize this transport. This quest requires detailed characterization of the transport, which is complicated by the fact that one usually lacks knowledge of the initial and final points of the transport process within the aggregate. First steps have been made by growing palladium nanoparticles on the tubular carbocyanine aggregates [62]. These particles act as quenchers, whose positions may be characterized by cryo-TEM. Also the growing possibilities of tip-enhanced optical excitation may be used in the future to create a well-defined excitation position on an aggregate.

\section{ACKNOWLEDGMENTS}

The author is grateful to his (former) coworkers V. A. Malyshev, M. Bednarz, C. Didraga, and D. J. Heijs and to his experimental colleagues K. Duppen, A. Pugžlys, P. H. M. van Loosdrecht, R. Augulis, S. Daehne, C. Spitz, S. Kirstein, and $H$. von Berlepsch. This work is part of the research program of the Stichting voor Fundamenteel Onderzoek der Materie (FOM), which is financially supported by the Nederlandse Organisatie voor Wetenschappelijk Onderzoek (NWO). Support was also received from NanoNed, a national nanotechnology program coordinated by the Dutch Ministry of Economic Affairs.

\section{REFERENCES}

[1] E. E. Jelley, "Spectral absorption and fluorescence of dyes in the molecular state," Nature, vol. 138, pp. 1009-1010, 1936.

[2] G. Scheibe, "Variability of the absorption spectra of some sensitizing dyes and its cause," Angewandte Chemie, vol. 49, p. 563, 1936.

[3] T. Tani, Photographic Sensitivity, Oxford University Press, Oxford, UK, 1995. 
[4] H. van Amerongen, L. Valkunas, and R. van Grondelle, Photosynthetic Excitons, World Scientific, Singapore, 2000.

[5] S. de Boer, K. J. Vink, and D. A. Wiersma, "Optical dynamics of condensed molecular aggregates: an accumulated photonecho and hole-burning study of the J-aggregate," Chemical Physics Letters, vol. 137, no. 2, pp. 99-106, 1987.

[6] H. Fidder, J. Knoester, and D. A. Wiersma, "Superradiant emission and optical dephasing in J-aggregates," Chemical Physics Letters, vol. 171, no. 5-6, pp. 529-536, 1990.

[7] H. Fidder and D. A. Wiersma, "Collective optical-response of molecular aggregates," Physica Status Solidi B-Basic Research, vol. 188, no. 1, pp. 285-295, 1995.

[8] E. O. Potma and D. A. Wiersma, "Exciton superradiance in aggregates: the effect of disorder, higher order exciton-phonon coupling and dimensionality," Journal of Chemical Physics, vol. 108, no. 12, pp. 4894-4903, 1998.

[9] V. F. Kamalov, I. A. Struganova, and K. Yoshihara, "Temperature dependent radiative lifetime of BIC J-aggregates," Journal of Physical Chemistry, vol. 100, no. 21, pp. 8640-8644, 1996.

[10] F. C. Spano, J. R. Kuklinski, and S. Mukamel, "Temperaturedependent superradiant decay of excitons in small aggregates," Physical Review Letters, vol. 65, no. 2, pp. 211-214, 1990.

[11] I. Renge and U. P. Wild, "Solvent, temperature, and excitonic effects in the optical spectra of pseudoisocyanine monomer and J-aggregates," Journal of Physical Chemistry Part A, vol. 101, no. 43, pp. 7977-7988, 1997.

[12] I. G. Scheblykin, O. Yu. Sliusarenko, L. S. Lepnev, A. G. Vitukhnovsky, and M. Van der Auweraer, "Excitons in molecular aggregates of 3,3'-Bis-[3-sulfopropyl]-5,5' -dichloro-9ethylthiacarbocyanine (THIATS): temperature dependent properties," Journal of Physical Chemistry Part B, vol. 105, no. 20, pp. 4636-4646, 2001.

[13] I. G. Scheblykin, O. Yu. Sliusarenko, L. S. Lepnev, A. G. Vitukhnovsky, and M. Van der Auweraer, "Strong nonmonotonous temperature dependence of exciton migration rate in J aggregates at temperatures from 5 to $300 \mathrm{~K}$," Journal of Physical Chemistry Part B, vol. 104, no. 47, pp. 10949-10951, 2000.

[14] A. V. Malyshev, V. A. Malyshev, and F. Domínguez-Adame, "Low-temperature quenching of one-dimensional localized Frenkel excitons," Chemical Physics Letters, vol. 371, no. 3-4, pp. 417-425, 2003.

[15] F. C. Spano and S. Mukamel, "Nonlinear susceptibilities of molecular aggregates: enhancement of $X^{(3)}$ by size," Physical Review A, vol. 40, no. 10, pp. 5783-5801, 1989.

[16] H. Ishihara and K. Cho, "Cancellation of size-linear terms in the third-order nonlinear susceptibility: frenkel excitons in a periodic chain," Physical Review B, vol. 42, no. 3, pp. 17241730, 1990.

[17] J. Knoester, "Third-order optical response of molecular aggregates. Disorder and the breakdown of size-enhancement," Chemical Physics Letters, vol. 203, no. 4, pp. 371-377, 1993.

[18] H. Fidder, J. Knoester, and D. A. Wiersma, "Observation of the one-exciton to two-exciton transition in a J aggregate," The Journal of Chemical Physics, vol. 98, no. 8, pp. 6564-6566, 1993.

[19] G. Juzeliunas, "Exciton absorption spectra of optically excited linear molecular aggregates," Zeitschrift für Physik D Atoms, Molecules and Clusters, vol. 8, no. 4, pp. 379-384, 1988.

[20] T. Meier, V. Chernyak, and S. Mukamel, "Multiple exciton coherence sizes in photosynthetic antenna complexes viewed by pump-probe spectroscopy," Journal of Physical Chemistry B, vol. 101, no. 37, pp. 7332-7342, 1997.

[21] L. D. Bakalis and J. Knoester, "Pump-probe spectroscopy and the exciton delocalization length in molecular aggregates," Journal of Physical Chemistry B, vol. 103, no. 31, pp. 66206628, 1999.

[22] A. M. van Oijen, M. Ketelaars, J. Köhler, T. J. Aartsma, and J. Schmidt, "Unraveling the electronic structure of individual photosynthetic pigment-protein complexes," Science, vol. 285, no. 5426, pp. 400-402, 1999.

[23] G. Scheibe, in Optische Anregungen Organischer Systeme, W. Först, Ed., p. 109, Chemie, Weinheim, Germany, 1966.

[24] G. Mc Dermott, S. M. Prince, A. A. Freer, et al., "Crystal structure of an integral membrane light-harvesting complex from photosynthetic bacteria," Nature, vol. 374 , no. 6522, pp. 517 521, 1995.

[25] J. Koepke, X. Hu, C. Muenke, K. Schulten, and H. Michel, “The crystal structure of the light-harvesting complex II (B800850) from Rhodospirillum molischianum," Structure, vol. 4, no. 5, pp. 581-597, 1996.

[26] L. A. Staehelin, J. R. Golecki, and G. Drews, "Supramolecular organization of chlorosomes (chlorobium vesicles) and of their membrane attachment sites in Chlorobium Limicola," Biochimica et Biophysica Acta (BBA)_Bioenergetics, vol. 589, no. 1, pp. 30-45, 1980.

[27] V. I. Prokhorenko, D. B. Steensgaard, and A. R. Holzwarth, "Exciton Dynamics in the Chlorosomal Antennae of the Green Bacteria Chloroflexus aurantiacus and Chlorobium tepidum," Biophysical Journal, vol. 79, no. 4, pp. 2105-2120, 2000.

[28] J. Pšenčík, T. P. Ikonen, P. Laurinmäki, et al., "Lamellar organization of pigments in chlorosomes, the light harvesting complexes of green photosynthetic bacteria," Biophysical Journal, vol. 87, no. 2, pp. 1165-1172, 2004.

[29] A. Pawlik, A. Ouart, S. Kirstein, H.-W. Abraham, and S. Daehne, "Synthesis and UV/Vis spectra of J-aggregating $5,5^{\prime}, 6,6^{\prime}$-tetrachlorobenzimidacarbocyanine dyes for artificial light-harvesting systems and for asymmetrical generation of supramolecular helices," European Journal of Organic Chemistry, vol. 2003, no. 16, pp. 3065-3080, 2003.

[30] H. von Berlepsch, C. Böttcher, A. Ouart, C. Burger, S. Daehne, and S. Kirstein, "Supramolecular structures of J-aggregates of carbocyanine dyes in solution," Journal of Physical Chemistry $B$, vol. 104, no. 22, pp. 5255-5262, 2000.

[31] H. von Berlepsch, C. Böttcher, A. Ouart, et al., "Surfactantinduced changes of morphology of J-aggregates: superhelixto-tubule transformation," Langmuir, vol. 16, no. 14, pp. 5908-5916, 2000.

[32] A. Pawlik, S. Kirstein, U. De Rossi, and S. Daehne, "Structural conditions for spontaneous generation of optical activity in Jaggregates," Journal of Physical Chemistry B, vol. 101, no. 29, pp. 5646-5651, 2000.

[33] S. Kirstein, H. von Berlepsch, C. Böttcher, et al., "Chiral J-aggregates formed by achiral cyanine dyes," Chemistry/Physical Chemistry, vol. 1, no. 3, pp. 146-150, 2000.

[34] C. Didraga, A. Pugžlys, P. R. Hania, H. von Berlepsch, K. Duppen, and J. Knoester, "Structure, spectroscopy, and microscopic model of tubular carbocyanine dye aggregates," Journal of Physical Chemistry B, vol. 108, no. 39, pp. 14976-14985, 2004.

[35] A. S. Davydov, Theory of Molecular Excitons, Plenum Press, New York, NY, USA, 1971.

[36] V. Czikkely, H. D. Försterling, and H. Kuhn, "Extended dipole model for aggregates of dye molecules," Chemical Physics Letters, vol. 6, no. 3, pp. 207-210, 1970.

[37] G. D. Scholes, I. A. Gould, R. J. Cogdell, and G. R. Fleming, "Ab initio molecular orbital calculations of eCouplings in the LH2 bacterial light-harvesting complex of Rps. acidophila," 
Journal of Physical Chemistry B, vol. 103, no. 13, pp. 25432553, 1999.

[38] H. Fidder, J. Knoester, and D. A. Wiersma, "Optical properties of disordered molecular aggregates: a numerical study," The Journal of Chemical Physics, vol. 95, no. 11, pp. 7880-7890, 1991.

[39] L. D. Bakalis, I. Rubtsov, and J. Knoester, "Absorption spectra of mixed two-dimensional cyanine aggregates on silver halide substrates," The Journal of Chemical Physics, vol. 117, no. 11, pp. 5393-5403, 2002.

[40] C. Didraga, J. A. Klugkist, and J. Knoester, "Optical properties of helical cylindrical molecular aggregates: the homogeneous limit," Journal of Physical Chemistry B, vol. 106, no. 44, pp. 11474-11486, 2002.

[41] A. Pugžlys, P. R. Hania, C. Didraga, J. Knoester, and K. Duppen, "Cylindrical aggregates of TDBC: linear and nonlinear optical properties versus morphology," Solid State Phenomena, vol. 97-98, pp. 201-206, 2004.

[42] A. Pugžlys, P. R. Hania, C. Didraga, V. A. Malyshev, J. Knoester, and K. Duppen, "Ultrafast exciton transport in organic nanotubes," in Ultrafast Phenomena XIV, T. Kobayashi, T. Okada, K. A. Nelson, and S. De Silvestri, Eds., p. 879, Springer, Berlin, Germany, 2005.

[43] C. Didraga and J. Knoester, "Chiral exciton wave functions in cylindrical J aggregates," The Journal of Chemical Physics, vol. 121, no. 2, pp. 946-959, 2004.

[44] M. Schreiber and Y. Toyozawa, "Numerical experiments on the absorption lineshape of the exciton under lattice vibrations. I. The overall lineshape," Journal of the Physical Society of Japan, vol. 51, no. 5, pp. 1528-1537, 1982.

[45] C. Didraga and J. Knoester, "Optical spectra and localization of excitons in inhomogeneous helical cylindrical aggregates," The Journal of Chemical Physics, vol. 121, no. 21, pp. 1068710698, 2004.

[46] V. A. Malyshev, "Localization length of a 1-D exciton and temperature dependence of the radiative lifetime in frozen dye solutions with J aggregates," Optics and Spectroscopy, vol. 71, no. 6, pp. 505-506, 1992.

[47] D. J. Heijs, V. A. Malyshev, and J. Knoester, "Decoherence of excitons in multichromophore systems: thermal line broadening and destruction of superradiant emission," Physical Review Letters, vol. 95, no. 17, Article ID 177402, 2005.

[48] E. W. Knapp, "Lineshapes of molecular aggregates, exchange narrowing and intersite correlation," Chemical Physics, vol. 85, no. 1, pp. 73-82, 1984.

[49] R. Hirschmann and S. Friedrich, "A hole burning study of excitonic states of chain molecules in glasses," The Journal of Chemical Physics, vol. 91, no. 12, pp. 7988-7993, 1989.

[50] M. Bednarz, V. A. Malyshev, and J. Knoester, "Intraband relaxation and temperature dependence of the fluorescence decay time of one-dimensional Frenkel excitons: the Pauli master equation approach," The Journal of Chemical Physics, vol. 117, no. 13, pp. 6200-6213, 2002.

[51] D. J. Heijs, V. A. Malyshev, and J. Knoester, "Thermal broadening of the J-band in disordered linear molecular aggregates: a theoretical study," The Journal of Chemical Physics, vol. 123, Article ID 144507, 12 pages, 2005.

[52] M. Shimizu, S. Suto, and T. Goto, "Theory and numerical study of exciton dynamics in a disordered linear chain," The Journal of Chemical Physics, vol. 114, no. 6, pp. 2775-2783, 2001.

[53] M. Bednarz, V. A. Malyshev, and J. Knoester, “Temperature dependent fluorescence in disordered Frenkel chains: interplay of equilibration and local band-edge level structure," Physical
Review Letters, vol. 91, no. 21, Article ID 217401, 2003.

[54] A. Pugžlys, R. Augulis, P. H. M. van Loosdrecht, C. Didraga, V. A. Malyshev, and J. Knoester, "Temperature-dependent relaxation of excitons in tubular molecular aggregates: fluorescence decay and Stokes shift," Journal of Physical Chemistry B, vol. 110, no. 41, pp. 20268-20276, 2006.

[55] M. Bednarz, V. A. Malyshev, and J. Knoester, "Lowtemperature dynamics of weakly localized Frenkel excitons in disordered linear chains," The Journal of Chemical Physics, vol. 120, no. 8, pp. 3827-3840, 2004.

[56] Th. Förster, "Zwischenmolekulare energie-wanderung und fluoreszenz," Annals of Physics, vol. 2, pp. 55-75, 1948.

[57] H. Sumi, "Theory on rates of excitation-energy transfer between molecular aggregates through distributed transition dipoles with application to the antenna system in bacterial photosynthesis," Journal of Physical Chemistry B, vol. 103, no. 1, pp. 252-260, 1999.

[58] K. Mukai, S. Abe, and H. Sumi, "Theory of rapid excitationenergy transfer from B800 to optically-forbidden exciton states of B850 in the antenna system LH2 of photosynthetic purple bacteria," Journal of Physical Chemistry B, vol. 103, no. 29, pp. 6096-6102, 1999.

[59] S. Jang, M. D. Newton, and R. J. Silbey, "Multichromophoric Förster resonance energy transfer," Physical Review Letters, vol. 92, no. 21, Article ID 218301, 2004.

[60] N. Fukutake, S. Takasaka, and T. Kobayashi, "Energy transfer between two kinds of J-aggregates studied by near-field absorption-fluorescence spectroscopy," Chemical Physics Letters, vol. 361, no. 1-2, pp. 42-48, 2002.

[61] C. Didraga, V. A. Malyshev, and J. Knoester, "Excitation energy transfer between closely spaced multichromophoric systems: Effects of band mixing and intraband relaxation ," Journal of Physical Chemistry B, vol. 110, no. 38, pp. 18818-18827, 2006.

[62] S. Kirstein, H. von Berlepsch, and C. Böttcher, "Photo induced reduction of noble metal ions to metal nonoparticles on tabular J-aggregates," International Journal of Photoenergy, vol. 2007, Article ID 47917, 2007. 


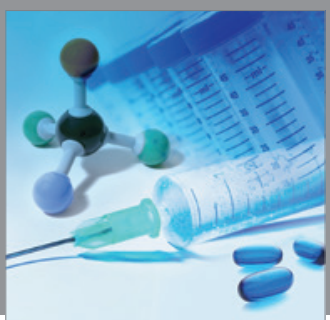

International Journal of

Medicinal Chemistry

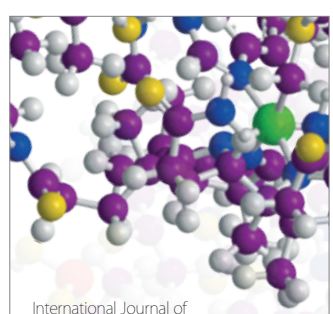

Carbohydrate Chemistry

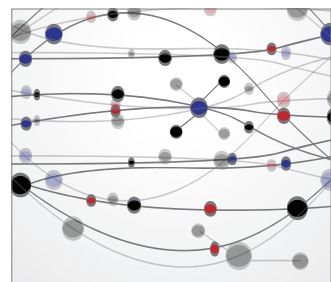

The Scientific World Journal
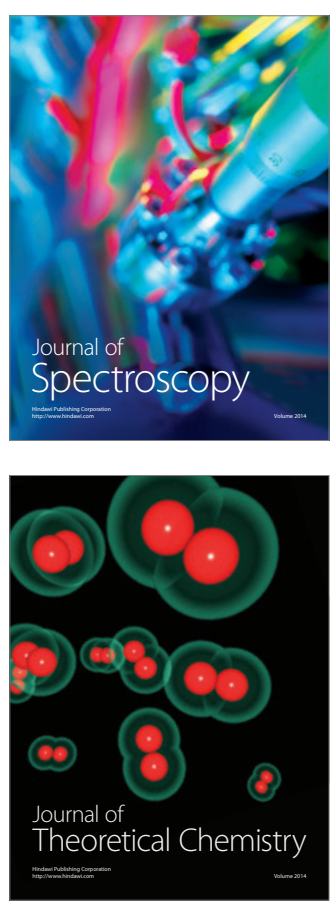
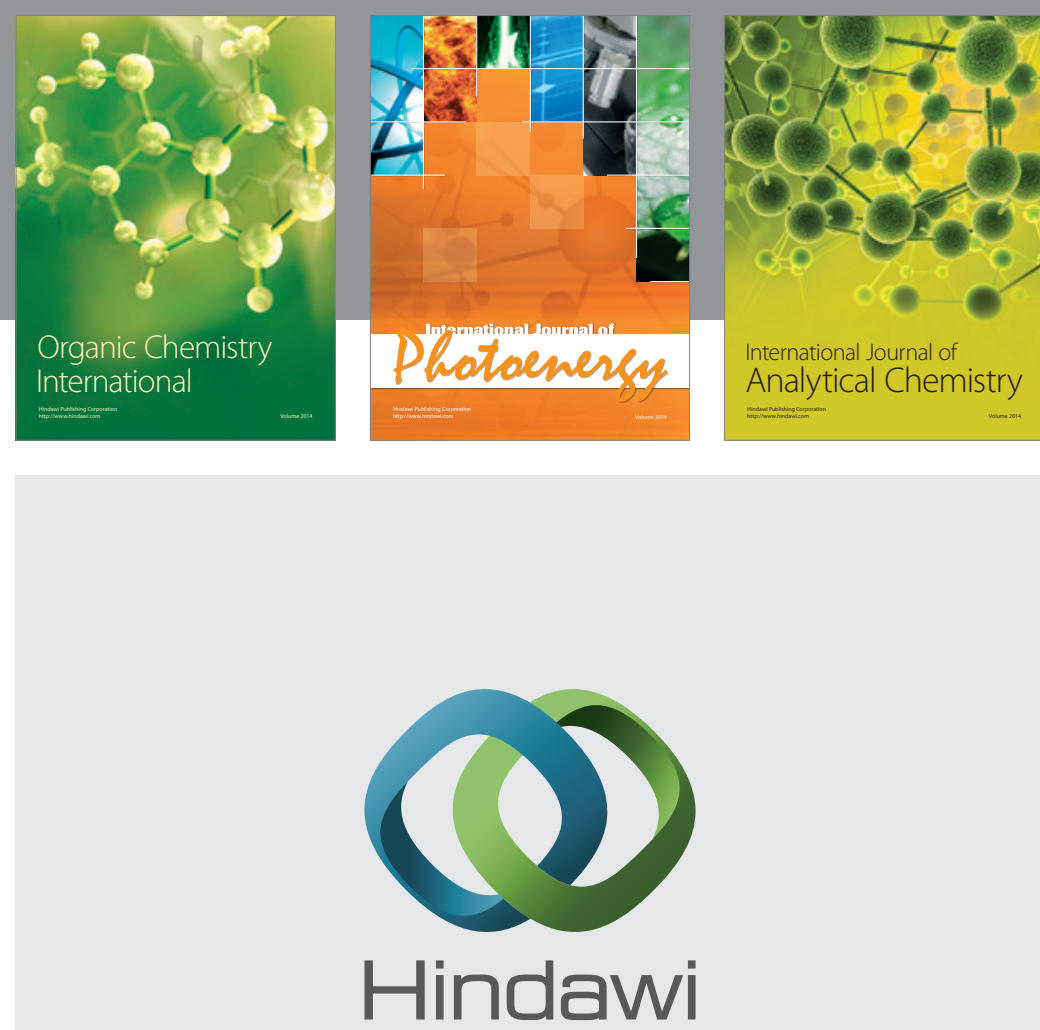

Submit your manuscripts at

http://www.hindawi.com
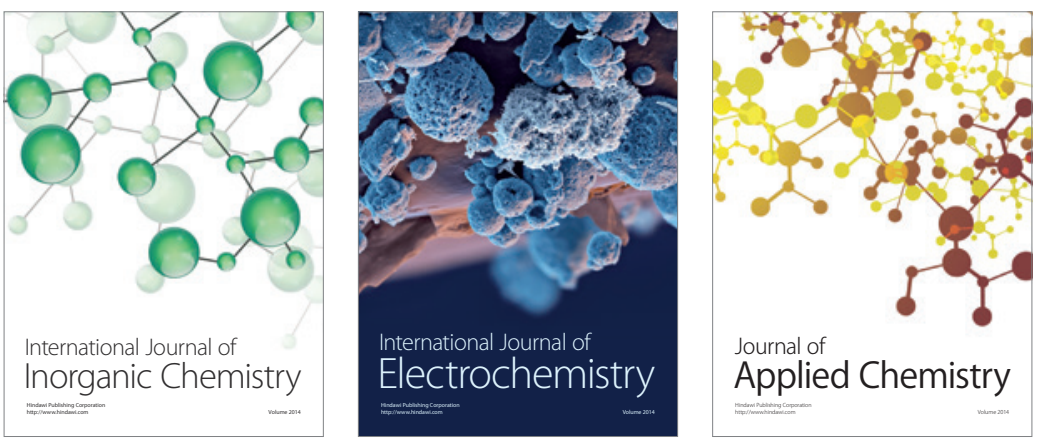

Journal of

Applied Chemistry
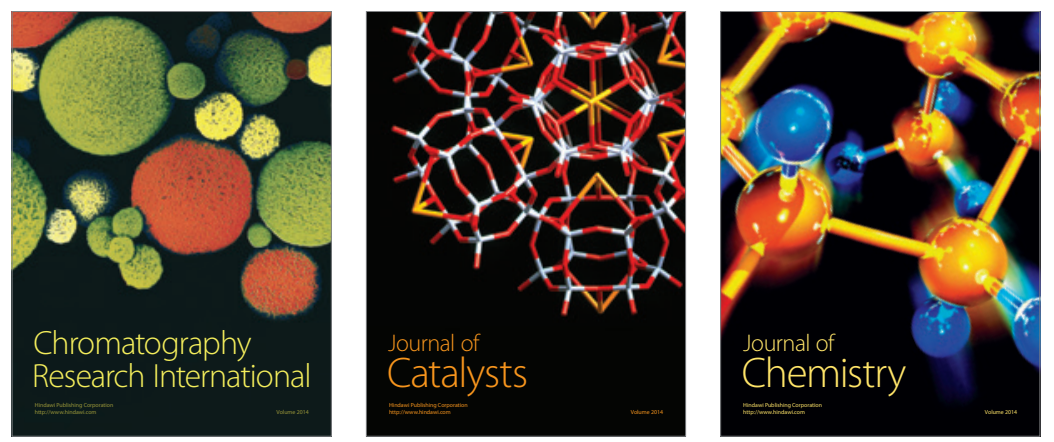
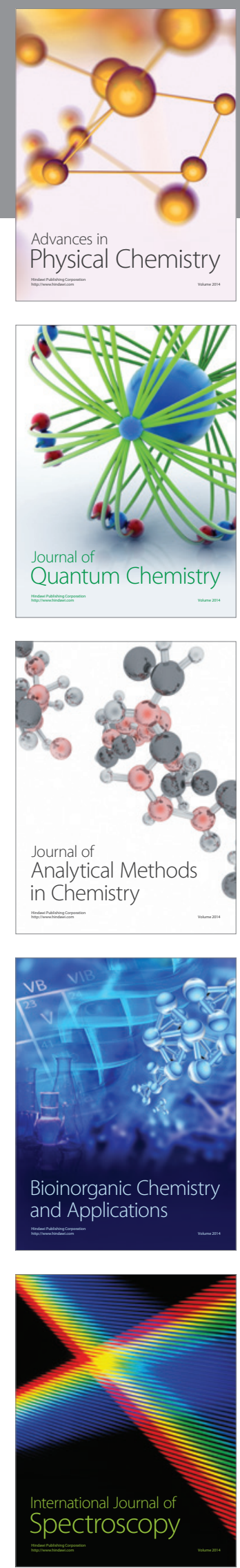